\title{
RESVERATROL INHIBITS EXPRESSION OF CANCER-SPECIFIC PENTOSE PHOSPHATE PATHWAY ENZYME TKTL1
}

\author{
BHUPENDER KUMAR ${ }^{1,2 *}$ \\ ${ }^{1}$ National Centre of Applied Human Genetics, School of Life Sciences, Jawaharlal Nehru University, New Delhi 110067, India. ${ }^{2}$ Department \\ of Biochemistry, Institute of Home Economics, University of Delhi, New Delhi 110016, India. Email: bhupender19@ihe.du.ac.in
}

Received: 30 January 2018, Revised and Accepted: 19 March 2018

\section{ABSTRACT}

Objective: The objective of this study was to identify cancer-specific metabolic enzyme Transketolase-like 1 (TKTL1) from pentose phosphate pathway as target of resveratrol, a naturally occurring nutraceutical.

Methods: Methylthiazolyldiphenyl-tetrazolium bromide assay and trypan blue assay were used for the estimation of growth and proliferation. Reactive oxygen species (ROS) was estimated using 2'-7'-Dichlorodihydrofluorescein diacetate while reduced glutathione (GSH) was estimated using commercially available kit. Promoter activity, reverse transcription polymerase chain reaction, and western blotting were used for expression analysis.

Results: Resveratrol treatment in HeLa cells inhibited proliferation, promoted ROS, and reduced intracellular GSH levels. In TKTL1 promoter activity assay, we found that resveratrol treatment directly inhibited promoter activity of TKTL1. Resveratrol inhibited both mRNA and protein expression of TKTL1 in a dose-dependent manner.

Conclusion: This is the first report where we show that resveratrol inhibits cancer-specific isoform TKTL1 as one of its targets to elicit its anticancer activity.

Keywords: Resveratrol, Transketolase-like 1, HeLa, Pentose phosphate pathway.

(C) 2018 The Authors. Published by Innovare Academic Sciences Pvt Ltd. This is an open access article under the CC BY license (http://creativecommons. org/licenses/by/4. 0/) DOI: http://dx.doi.org/10.22159/ajpcr.2018.v11i6.25021

\section{INTRODUCTION}

Transketolase-like 1 (TKTL1) is an isoform of pentose phosphate pathway (PPP) enzyme TKT, usually found overexpressed in different cancer types due to promoter hypomethylation [1]. TKTL1 overexpression or knockdown has been shown to modulate the amount of ribose-5-phosphate (R5P), the precursor molecule for nucleotide synthesis [2]. TKTL1 has been shown to induce Warburg effect and aerobic glycolysis, thereby providing raw materials required for rapid proliferation of cancer cells [3-5]. Since TKTL1 expression is found to be exclusively upregulated in different cancers, it is also proposed as a marker for predicting outcomes [6,7]. Ectopic expression or inhibition of TKTL1 has been shown to affect proliferation of cancer cells both in vitro and in vivo $[8,9]$.

Resveratrol (3, 5, 4'-trihydroxystilbene) is a well-known phytoalexin shown to possess anticancer, antiobesity, and antidiabetic properties [10-12]. A number of mechanisms underlying the anticancer property of resveratrol have been reported [13,14]. Recent studies have demonstrated that resveratrol targets cancer cells by inhibiting many important metabolic enzymes such as those involved in glucose metabolism [15-17]. The present study unravels another metabolic enzyme TKTL1 as a target of resveratrol. The present study reports how resveratrol inhibits TKTL1 expression in a dose-dependent manner where the inhibition was seemingly through decreasing its promoter activity, leading to both reduced mRNA and corresponding amount of protein.

\section{METHODS}

\section{Cell culture and drug treatment}

HeLa cells were maintained as described previously [18]. For drug treatment, $24 \mathrm{~h}$ before treatment, 0.2 million cells were seeded in 6-well plates (Corning). Next day cells were treated with drug solvent and resveratrol for $48 \mathrm{~h}$. Ethanol (solvent)-treated cells were used as control.
Drug treatment, cellular viability, and proliferation studies Cell viability and cellular proliferation were assessed using methylthiazolyldiphenyl-tetrazolium bromide (Sigma) and trypan blue as described previously [16].

Reactive oxygen species (ROS) and reduced glutathione (GSH) estimation

Intracellular ROS and GSH levels were estimated as described previously [16].

Promoter activity assay, mRNA isolation, and reverse transcription polymerase chain reaction (RT-PCR)

TKTL1 promoter $(1.2 \mathrm{~kb})$ was cloned in pGL3 luciferase reporter vector (Promega, USA). HeLa cells were seeded at a density of $1 \times 10^{5}$ cells per well in a 6-well plate 1 day before transfection. A total of $1 \mu \mathrm{g}$ reporter vector and $0.1 \mu \mathrm{g}$ of pRL-TK Renilla luciferase vector (Promega, USA) were transfected with lipofectamine LTX reagent (Invitrogen, USA), which was followed by resveratrol treatment. Cotransfected pRLTK vector was used as an internal control to normalize transfection efficiency. After $48 \mathrm{~h}$ of treatment, cells were harvested and luciferase activity detected using luminometer (TD-20/20, DLReady; Turner Designs, Promega, USA). Total RNA from treated cells was isolated using TRIzoL reagent (Sigma, USA) as per manufacturer's guidelines. The concentration of extracted RNA was measured by taking absorbance at $260 / 280 \mathrm{~nm}$ on nanodrop and by checking on a $1.2 \%$ formaldehyde agarose gel. 1-2 $\mu$ g of total RNA was converted to single-stranded cDNA using RT enzyme (Applied Biosystems, USA). RT-PCR was performed for mRNA analysis of TKTL1 while beta-actin was used as internal control. Primer sequences used for cloning of TKTL1 promoter and for performing RT-PCR are provided in Table 1.

Cell lysate preparation, protein estimation, and western blotting Cell lysate preparation, protein estimation, and western blotting were performed as described previously [16]. Primary antibodies used were: 


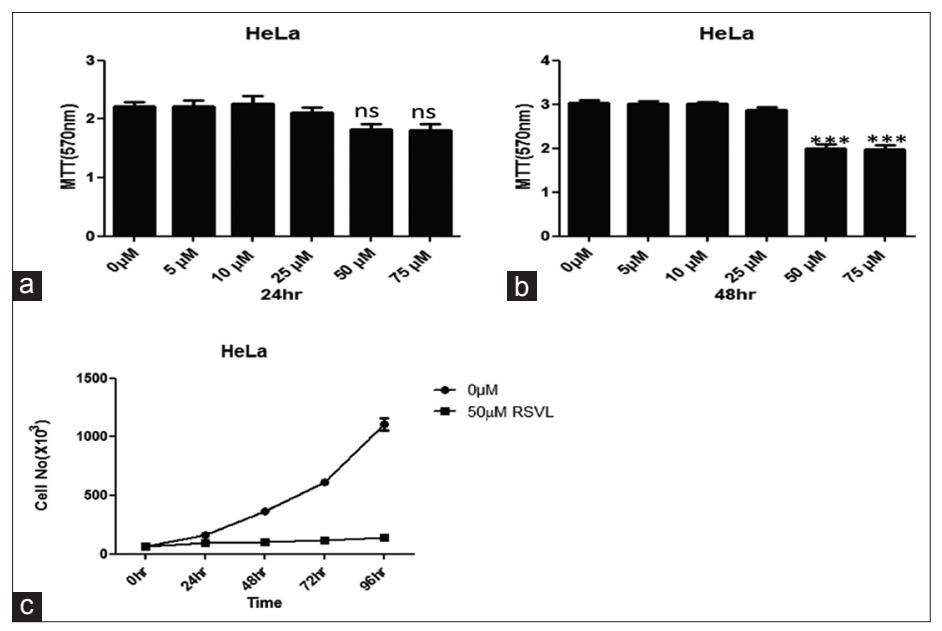

Fig. 1: Resveratrol inhibits viability and proliferation of HeLa cancer cells. (a) Viability of HeLa cells was estimated by methylthiazolyldiphenyl-tetrazolium bromide assay following treatment with different concentration of resveratrol for $24 \mathrm{~h}$; (b) for $48 \mathrm{~h}$. (c) Cellular proliferation was estimated by cell counting following treatment with $50 \mu \mathrm{M}$ resveratrol for different time intervals. All data represented as means $\pm \mathrm{SE}, \mathrm{n}=3,{ }^{* * *} \mathrm{p}<0.001$

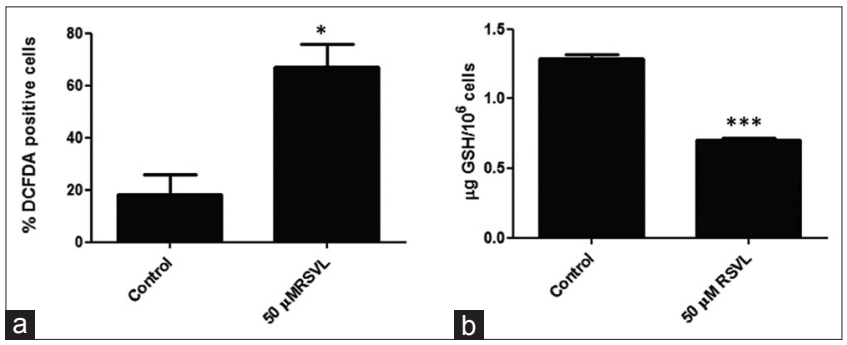

Fig. 2: Resveratrol treatment promotes reactive oxygen species (ROS) by reducing intracellular glutathione (GSH) levels. (a) Intracellular levels of ROS were assessed using 2'-7'-Dichlorodihydrofluorescein diacetate dye following treatment with $50 \mu \mathrm{M}$ resveratrol for $48 \mathrm{~h}$ in HeLa cells.

(b) Intracellular GSH (mg/106 cells) in HeLa cells after treatment with resveratrol. All data represented as means $\pm S E, n=3,{ }^{*} p<0.05$, ${ }^{* * * *} \mathbf{p}<0.001$

Anti-TKTL1 (Abcam) and anti- $\beta$-actin (Cell Signaling Technology). HRP conjugated corresponding secondary antibodies (Cell Signaling Technology, USA) were used.

\section{RESULTS}

Resveratrol inhibits viability and proliferation of HeLa cancer cells To identify the effective dose of inhibition, HeLa cells were treated with different concentrations of resveratrol for $24 \mathrm{~h}$ and $48 \mathrm{~h}$. Treatment of HeLa cells with resveratrol resulted in a dose-dependent decrease in cellular viability where $24 \mathrm{~h}$ treatment resulted in a non-significant decrease (Fig. 1a) while $48 \mathrm{~h}$ treatment resulted in a statistically significant decrease at $50 \mu \mathrm{M}$ and above dose (Fig. 1b). As $50 \mu \mathrm{M}$ dose showed significant inhibition, this dose was used to study the effect of resveratrol on proliferation of HeLa cells till 96 h. HeLa cells treated with solvent showed increase in cell number for different time durations while cells treated with resveratrol showed relatively no increase in cell number (Fig. 1c). Interestingly, complete inhibition of cells was not observed even at $96 \mathrm{~h}$.

Resveratrol promotes intracellular ROS by decreasing GSH levels in HeLa cells

Intracellular reduced GSH is primarily responsible for keeping ROS levels under a check. Increase in ROS above a threshold level would, therefore, be detrimental for a cancer cell. Resveratrol treatment in HeLa cells resulted in increased intracellular ROS levels (Fig. 2a). To confirm
Table 1: Primer sequences used for TKTL1

\begin{tabular}{|c|c|c|}
\hline Primer & Sequence & $\begin{array}{l}\text { Amplicon } \\
\text { size (bp) }\end{array}$ \\
\hline \multicolumn{3}{|l|}{$\begin{array}{l}\text { For promoter } \\
\text { cloning }\end{array}$} \\
\hline FP & $\begin{array}{l}\text { 5'-GAGAGGTACC } \\
\text { CTGCCTCAGATTCCCATTAG-3' }\end{array}$ & 1200 \\
\hline $\mathrm{RP}$ & $\begin{array}{l}\text { 5'-GAGAAGATCT } \\
\text { TGGAATGTTTGTGACTTGCAT-3 }\end{array}$ & \\
\hline \multicolumn{3}{|l|}{ For RT-PCR } \\
\hline RT-TKTL1-FP & 5'-GCTGAACAAAACATGGTGAGC-3' & 310 \\
\hline RT-TKTL1-RP & 5'-ACATCCCCTTGGCATTGGCT-3' & \\
\hline RT-actin-FP & 5'-ACTCTTCCAGCCTTCCTTC-3' & 171 \\
\hline RT-actin-RP & 5'-АTCTCCTTCTGCATCCTGTC-3' & \\
\hline
\end{tabular}

the involvement of GSH intracellular, GSH levels were also checked. Resveratrol treatment also resulted in reduction of intracellular GSH level (Fig. 2b). Since NADPH produced exclusively in PPP is utilized for synthesis of GSH, involvement of a PPP enzyme was thought pertinent.

Resveratrol treatment inhibits TKTL1 expression through inhibition of its promoter activity

To understand the growth inhibitory effect of resveratrol on HeLa cells, we assessed the involvement of cancer-specific PPP enzyme TKTL1, whose expression is known to promote proliferation of cancer cells [3]. First, we assessed TKTL1 promoter activity in the presence and absence of resveratrol in HeLa cells. Resveratrol treatment resulted in $75 \%$ reduction of promoter activity (Fig. 3a). To confirm the effect of reduced promoter activity on TKTL1 mRNA production, RT-PCR was performed, where corresponding reduction of TKTL1 mRNA was observed (Fig. 3b). Finally, western blotting was performed which corroborated with the previous result, and a dose-dependent decrease in protein levels of TKTL1 was observed due to resveratrol treatment (Fig. 3c). As observed with RT-PCR and western blotting results, $25 \mu \mathrm{M}$ resveratrol treatment for $48 \mathrm{~h}$ was sufficient to affect the expression of TKTL1 in HeLa cells.

\section{DISCUSSION}

Resveratrol is well-known to possess anticancer properties; however, it is never been associated with TKTL1, a metabolic enzyme of PPP. Its relation with metabolic enzymes which provide selective advantage to cancer cells remains elusive. Although some researchers have shown that resveratrol affects important metabolic enzymes such as 


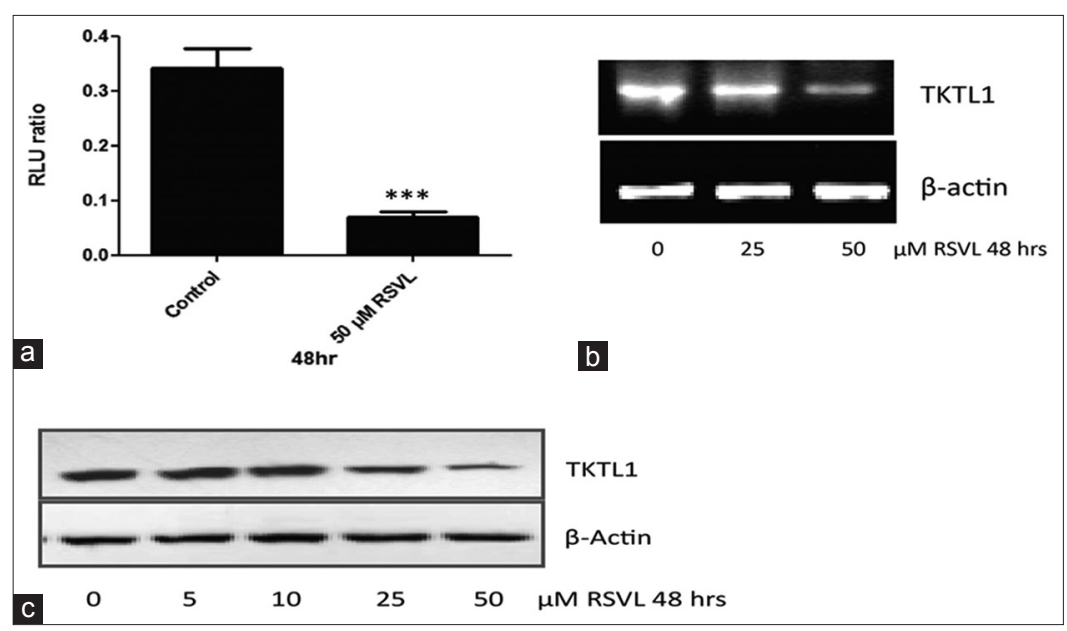

Fig. 3: Resveratrol inhibits promoter activity, mRNA, and protein level of transketolase-like 1 (TKTL1). (a) TKTL1 promoter activity estimated by dual luciferase assay on treatment with $50 \mu \mathrm{M}$ resveratrol for $48 \mathrm{~h}$. (b) Dose-dependent inhibition of TKTL1 mRNA levels estimated using reverse transcription polymerase chain reaction from HeLa cells treated with different concentrations of resveratrol for $48 \mathrm{~h}$. (c) Dose-dependent reduction of TKTL1 protein estimated using western blotting from HeLa cells. Data represented as means \pm SE, $\mathbf{n}=3, * * * \mathbf{p}<0.001$

phosphofructokinase, pyruvate kinase M2 (PKM2), and TP53-induced glycolysis and apoptosis regulator (TIGAR) [15-17], till date, no one reported any effect of resveratrol on TKTL1. This study for the first time shows that resveratrol directly inhibits mRNA and protein levels of TKTL1. TKTL1 is an enzyme known to promote nucleotide synthesis by providing the precursor molecule ribose-5-phosphate [19]. Metabolic reprogramming and increased glucose utilization, leading to Warburg effect is hallmark of cancer cells [20]. This increased glycolytic rate results in increased glycolytic intermediates which are channeled into non-oxidative and oxidative branches of PPP, deregulation of which dramatically impacts cancer cell proliferation [21]. TKTL1 utilizes these channeled intermediates and converts them into precursor material for nucleotide synthesis, thereby promoting anabolism in cancer cells. For sustaining rapid proliferation (DNA replication), cancer cells require enormous amount of these nucleotides and amino acids; therefore, the dependency of cancer cells on metabolic enzymes which helps in the production of these basic materials become very crucial. Multiple factors in cancer cells lead to increased ROS production, to keep the ROS levels under non-damaging levels cells produce reducing equivalents such as GSH [22]. GSH is exclusively synthesized by utilizing NADPH produced from the oxidative branch of PPP; therefore, PPP and its enzymes become very crucial for survival of cancer cells. Metabolic enzymes such as PKM2 and TKTL1 are selectively overexpressed in cancer cells; therefore, inhibition of these enzymes by resveratrol will selectively affect cancer cells. This study reports how resveratrol inhibits proliferation of cancer cells by increasing ROS levels and by targeting cancer-specific enzyme TKTL1.

Previously, our group reported that resveratrol targets PKM2 and TIGAR to affect the levels of ROS and glycolytic intermediates which leads to induction of autophagy and cell death of cancer cells by apoptosis [14,15]. This study elucidated how resveratrol targets PPP by inhibiting TKTL1 to further affect cancer cell proliferation. Interestingly, promoter activity data also suggest involvement of some transcription factors which normally promotes high expression of this cancer-promoting metabolic enzyme, but in the presence of resveratrol, they are not able to promote expression of these enzymes. It may be postulated that these upstream transcription factors might have been modified or downregulated in the presence of resveratrol leading to the observations found in this study.

\section{CONCLUSION}

This study reveals TKTL1 as an important target of resveratrol and also proposes a detailed future study to find the effect of resveratrol on important metabolic enzymes from different pathways to fully understand its mechanism of action.

\section{ACKNOWLEDGMENT}

BK acknowledges NCAHG, SLS, and JNU, where part of the work was carried out, for providing cell lines and regents as well.

\section{AUTHOR'S CONTRIBUTION}

BK conceived, designed, and performed the experiments. BK also did data analysis and manuscript writing.

\section{CONFLICT OF INTEREST}

The authors declare no competing interest.

\section{REFERENCES}

1. Sun W, Liu Y, Glazer CA, Shao C, Bhan S, Demokan S, et al. TKTL1 is activated by promoter hypomethylation and contributes to head and neck squamous cell carcinoma carcinogenesis through increased aerobic glycolysis and HIF1alpha stabilization. Clin Cancer Res 2010;16:857-66.

2. Dong Y, Wang M. Knockdown of TKTL1 additively complements cisplatin-induced cytotoxicity in nasopharyngeal carcinoma cells by regulating the levels of NADPH and ribose-5-phosphate. Biomed Pharmacother 2017;85:672-8.

3. Li J, Zhu SC, Li SG, Zhao Y, Xu JR, Song CY, et al. TKTL1 promotes cell proliferation and metastasis in esophageal squamous cell carcinoma. Biomed Pharmacother 2015;74:71-6.

4. Hu LH, Yang JH, Zhang DT, Zhang S, Wang L, Cai PC, et al. The TKTL1 gene influences total transketolase activity and cell proliferation in human colon cancer loVo cells. Anticancer Drugs 2007;18:427-33.

5. Langbein S, Zerilli M, Zur Hausen A, Staiger W, Rensch-Boschert K, Lukan N, et al. Expression of transketolase TKTL1 predicts colon and urothelial cancer patient survival: Warburg effect reinterpreted. Br J Cancer 2006;94:578-85.

6. Song Y, Liu D, He G. TKTL1 and p63 are biomarkers for the poor prognosis of gastric cancer patients. Cancer Biomark 2015;15:591-7.

7. Grimm M, Munz A, Teriete P, Nadtotschi T, Reinert S. GLUT-1(+)/ TKTL1(+) coexpression predicts poor outcome in oral squamous cell carcinoma. Oral Surg Oral Med Oral Pathol Oral Radiol 2014:117:743-53.

8. Zhang S, Yang JH, Guo CK, Cai PC. Gene silencing of TKTL1 by RNAi inhibits cell proliferation in human hepatoma cells. Cancer Lett 2007:253:108-14

9. Yuan W, Wu S, Guo J, Chen Z, Ge J, Yang P, et al. Silencing of TKTL1 by siRNA inhibits proliferation of human gastric cancer cells in vitro 
and in vivo. Cancer Biol Ther 2010;9:710-6.

10. Jang M, Cai L, Udeani GO, Slowing KV, Thomas CF, Beecher CW, et al. Cancer chemopreventive activity of resveratrol, a natural product derived from grapes. Science 1997;275:218-20.

11. Mohamed H. Anti-obesity effect of resveratrol in rats on high fat diet through regulation of gene expression of visceral white adipose tissue. Int J Pharm Pharm Sci 2016;8:378-384.

12. Lalitha V, Korah MC, Sengottuvel S, Sivakumar T. Antidiabetic and antioxidant activity of resveratrol and vitamin-c combination on streptozotocin induced diabetic rats. Int J Pharm Pharm Sci 2015;7:455-8

13. Lin JN, Lin VC, Rau KM, Shieh PC, Kuo DH, Shieh JC, et al. Resveratrol modulates tumor cell proliferation and protein translation via SIRT1dependent AMPK activation. J Agric Food Chem 2010;58:1584-92.

14. Quoc Trung L, Espinoza JL, Takami A, Nakao S. Resveratrol induces cell cycle arrest and apoptosis in malignant NK cells via JAK2/STAT3 pathway inhibition. PLoS One 2013;8:e55183.

15. Iqbal MA, Bamezai RN. Resveratrol inhibits cancer cell metabolism by down regulating pyruvate kinase M2 via inhibition of mammalian target of rapamycin. PLoS One 2012;7:e36764.
16. Kumar B, Iqbal MA, Singh RK, Bamezai RN. Resveratrol inhibits TIGAR to promote ROS induced apoptosis and autophagy. Biochimie 2015;118:26-35

17. Gomez LS, Zancan P, Marcondes MC, Ramos-Santos L, MeyerFernandes JR, Sola-Penna M, et al. Resveratrol decreases breast cancer cell viability and glucose metabolism by inhibiting 6-phosphofructo-1kinase. Biochimie 2013;95:1336-43.

18. Kumar B, Bamezai RN. Moderate DNA damage promotes metabolic flux into PPP via PKM2 Y-105 phosphorylation: A feature that favours cancer cells. Mol Biol Rep 2015;42:1317-21.

19. Diaz-Moralli S, Aguilar E, Marin S, Coy JF, Dewerchin M, Antoniewicz MR, et al. A key role for transketolase-like 1 in tumor metabolic reprogramming. Oncotarget 2016;7:51875-97.

20. Vander Heiden MG, Cantley LC, Thompson CB. Understanding the warburg effect: The metabolic requirements of cell proliferation. Science 2009;324:1029-33

21. Jiang P, Du W, Wu M. Regulation of the pentose phosphate pathway in cancer. Protein Cell 2014;5:592-602.

22. Trachootham D, Lu W, Ogasawara MA, Nilsa RD, Huang P. Redox regulation of cell survival. Antioxid Redox Signal 2008;10:1343-74. 